\title{
Transforming Medical Advice Into Clinical Activities for Patient Follow-up
}

\author{
António Silva ${ }^{1}$, Tiago Oliveira ${ }^{2}$, José Neves ${ }^{1}$, Paulo Novais ${ }^{1}$ \\ 1 Algoritmi Centre/Department of Informatics, University of Minho, Braga, Portugal \\ id67830alunos.uminho.pt \\ \{pjon, jneves\}@di.uminho.pt \\ 2 National Institute of Informatics, Tokyo, Japan \\ toliveira@nii.ac.jp
}

\begin{abstract}
The delivery of Computer-Interpretable Guidelines occurs mainly in the form of tools following a Q\&A strategy for the interaction with users. Despite the interactivity conferred by this style of communication, currently available tools do not possess control mechanisms to ensure the fulfilment of clinical recommendations. With the CompGuide personal assistant web application for scheduling clinical tasks, besides the normal input of information and the production of recommendations by an execution engine, there is a mapping of these recommendations to an agenda of activities for the health care professional. These activities are also monitored and notifications are produced to ensure their delivery times.
\end{abstract}

\section{Introduction}

Clinical Practice Guidelines (CPGs) are systematically developed statements that provide clinical advice for specific circumstances. Their representation and deployment in Clinical Decision Support Systems (CDSSs) confers them the designation of Computer-Interpretable Guidelines (CIGs) [10]. Existing tools specialized in CIG-based advice follow a style of communication that resembles a digital Q\&A, in which a recommendation is produced, followed by a question to obtain information about the state of the patient, then followed by another recommendation. They possess no mechanisms to incentivize users to put recommendations in practice and do not monitor their execution [3]. As is known, monitoring patients is a laborious task due to the number of patients that a health care professional has to take care of and the complexity of procedures to be applied. Although CDSSs for patient-specific advice may help to ease this burden, the simple provision of recommendations may prove to be insufficient to generate a positive impact in the outcomes of care [6].

There is a growing need for CDSSs that, along with their patient-specific advice, also provide functionalities that would allow them to become more prominent in daily clinical practice, specially when it comes to patient tracking, patient follow-up, scheduling of procedures, and monitoring of procedure constraints. At 
the same time, it is important for these functionalities to be available in any setting, at any time, so that clinical advice can reach the health care professional in the most diverse situations.

The CompGuide framework is based on digital versions of clinical protocols for automatic interpretation. The application performs the role of a personal assistant for health care professionals that provides decision support and treatment recommendations.

Thus, the present work based on the CompGuide framework, proposes a new method for the integration of the advice of CPGs in the daily life of health personnel, such as physicians and nurses. The underlying principle is mapping clinical recommendations, with their respective temporal constraints, to an agenda of activities for a health care professional to perform.

The present paper is organized as follows. Section 2 describes related work regarding tools for CIG execution, along with a description of their current drawbacks and aspects to improve. Section 3 presents the CompGuide architecture for the deployment of CIGs and how its services are used as a basis for the health care assistant tool developed to accompany health care professionals. Section 4 describes the main elements of the personal assistant web application and its main functionalities. Finally, Section 7 presents the conclusions drawn so far with the development of the health care assistant and future directions for the work.

\section{Related Work}

Following the existence of numerous approaches to CIG modelling, there is an equal number of tools to operationalize CIGs and make them executable against patient data. This is the case of CIG execution engines such as GLEE [14], the Spock Engine [15], the GLARE Execution Engine [13], or SAGEDesktop [1], which were specifically developed for the application of guidelines to patients in health care settings. Their objective is to run CIG instructions against data from patients and produce tailored recommendations, according to the observed state. In these systems, the role of the execution engine is straightforward, in the sense that it is merely concerned with following the constraints of the clinical careflow, comparing items of the patient state with conditions stated in rules dictating whether a recommendation should be provided or not. Most tools for CIG execution, including the above-given examples, exist in the form of client-server applications, with the intelligence engine placed on the client side. Furthermore, these applications are mostly available as desktop applications, which is an obstacle to their potential for reaching health care professionals and their ease of deployment.

The idea of enhancing CDSSs with additional features that allow them to achieve a higher level of integration of clinical recommendations in clinical practice comes from the ever-increasing role of Ambient Assisted Living (AAL) in enabling new information and communication services which transparently support people in their everyday lives $[7,5]$. In fact, a similar idea has been explored 
in [2], where a personal memory assistant, capable of intelligent scheduling and deployed over a platform called iGenda. The assistant acts as the support for a centralized manager system that can manage several services and is responsible for the scheduling of multiple agendas, taking into account the availability of resources or the health conditions of the users. However, here the agenda manager is directed towards patients, which are, predominantly elderly people. Although different, the work proposed herein can be related to this project and to others such as the Collaborative Memory Aids [11] and Hermes [4], but with the focus placed on the health care professional.

\section{CompGuide Architecture for CIG Execution}

The CompGuide architecture, shown in Figure 1 is service-oriented and provides the functionalities of an execution engine. The Core Server is the central component of the architecture and was developed as a RESTful service application. The usage of web services as the means to access the Core Server offers expandability and the possibility to improve selected services without compromising others. This grants greater flexibility when integrating CIG execution functionalities in third party applications [8].

The Core Server has four modules. The first is the Authentication Agent which provides authentication and authorization to the different types of users of the system, namely administrators, health care professionals, or patients. The Guideline Handler is responsible for managing updates to the Guideline Repository, keeping different CIGs represented according to the CompGuide ontology [9], organized by authorship and by date. In order to use a guideline for execution, the Guideline Handler accesses the selected CIG in the Guideline Repository and pulls the corresponding careflow, delivering it to the Guideline Execution Engine, which, in turn, uses information about the patient state provided by the Database Handler to fill in the data entry points of the careflow and produce recommendations. The Guideline Execution Engine interprets all the scheduling constraints on the tasks and controls enactment times, which means that starting and ending times are verified and delivered to other applications. These mechanisms to follow the application of procedures over time and to verify the execution of tasks are absent from most CIG frameworks [12], but they are essential to have a decision support that is truly capable of following up on the application of a CPG.

These features are made available by the Core Server as RESTful web services in order to ensure they can be easily integrated into any type of application. The Core Server is implemented in Java, using the RESTEasy API over a WildFly Application Server. The personal assistant, which uses the web services available in the CoreServer, was developed as a web application following the Model-ViewControl(MVC) paradigm using Java Server Faces (JSF). The purpose of the Core Server is to make available CIG services that anyone can integrate in their own applications, with a special focus on AAL applications. 


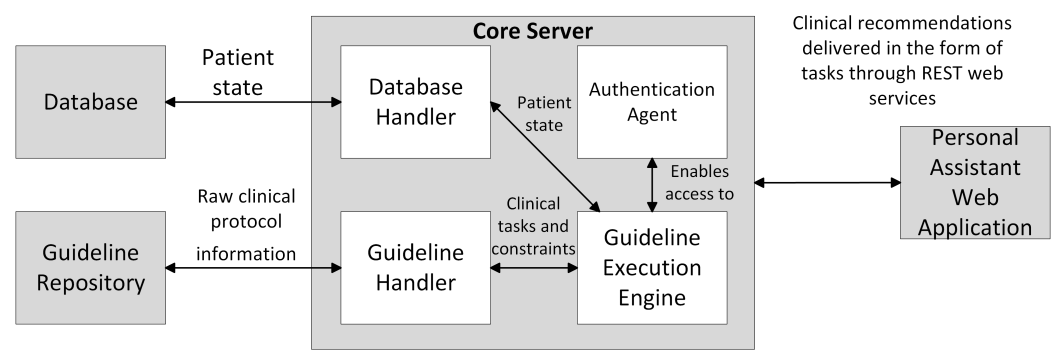

Fig. 1: Architecture of CompGuide Server Application.

\section{Health Care Personal Assistant Web Application}

The overall objective of the introduction of CPGs in decision support systems is to lead to a better acceptance and application of these good medical practices, since these systems are able to monitor the actions and observations of health care professionals. By using information and communication technology, it is possible to provide CIGs with a dynamism, presence, and interactivity that may bring them closer to the concept of living guidelines, which conveys a greater impact of good practices in health care, while still allowing the adaptation of these good practices to particular settings and situations.

In order to achieve this, it is necessary to develop a tool that not only allows the management of patients and the search for a CPG to apply, but also to control a number of circumstances of CIG execution, namely their execution times. That is the purpose of the CompGuide personal assistant web application.

\section{Main Elements of the Application}

In the application, it is possible for health care professionals to visualize the clinical tasks provided by the execution engine. This visualization can take one of two forms. The first is a timeline in which all the clinical tasks are displayed over a chronogram. A timeline of activities has the ability to compress multiple tasks into a single continuity that makes the succession of clinical procedures easy to understand. The benefits from such a representation include the ability to sequence events and reduce the potential for overburdening the health care professional. Additionally, by visualizing all of the pieces of a guideline treatment, care providers can make more focused, effective decisions about resources and timetables. This view is shown in Figure 2. In it, it is possible to observe clinical tasks for the management of colon cancer, namely sequential workup actions to ascertain the state of the patient.

The other available view is a calendar in which the health care professional can visualize the tasks according to the temporal granularity he sees fit, namely week, day, and month. While with the timeline it is easier to detect the starting 


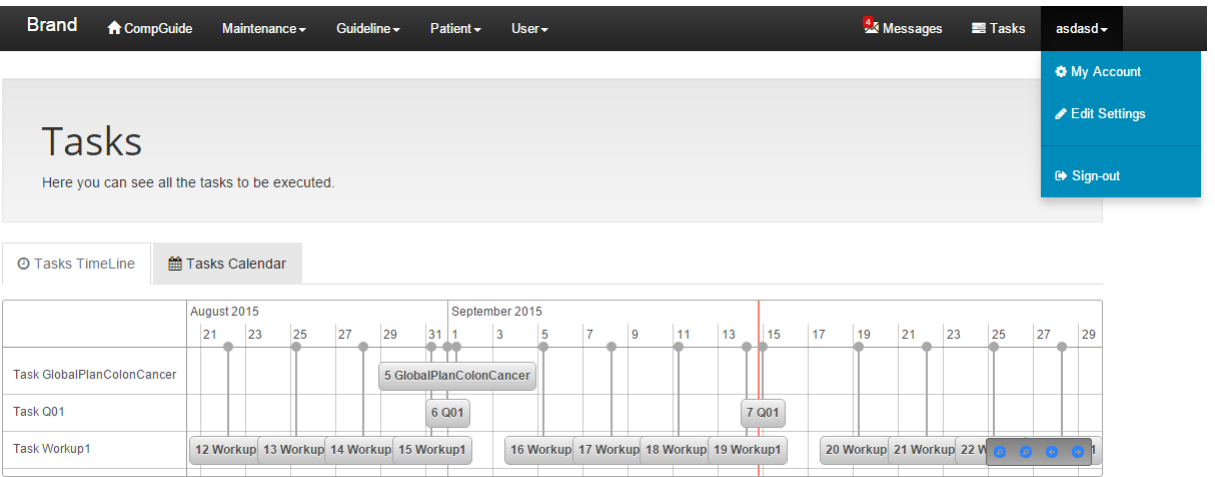

Fig. 2: Timeline view of clinical procedures in the CompGuide personal assistant web application.

and ending points of tasks, with the calendar view it is easier to grasp the temporal constraints that bind clinical tasks such as durations, waiting times and periodicities. Figure 3 shows the same tasks as in the timeline, but displayed over a week, where it is possible to verify, for instance, how long a clinical task should be applied.

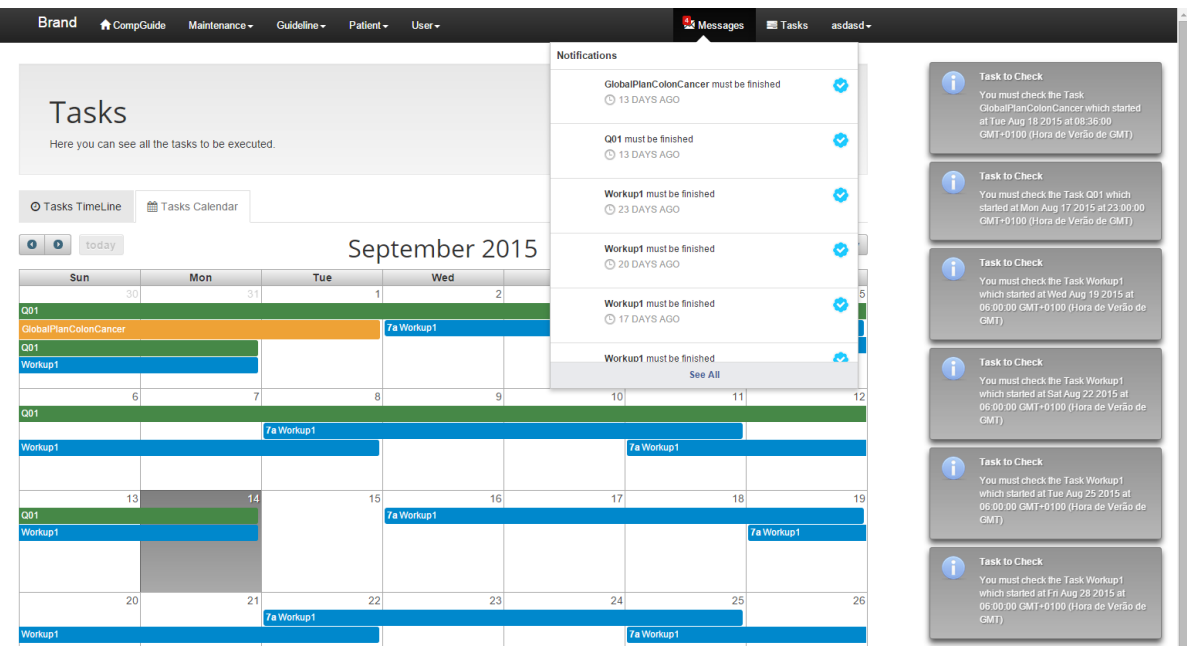

Fig. 3: Calendar view of clinical procedures in the CompGuide personal assistant web application. 


\section{Functionalities Supporting Care}

The CompGuide personal assistant web application makes it possible to manage information about CPGs, health care professionals that are users in the system, and patients to which CPGs are applied. As such, one can create, edit and delete all this information, according to the type of authorization in the system.

The Execution Engine from the CompGuide architecture was integrated in the tool in order to interpret CIGs in the repository and produce inferences that ultimately result in recommendations of clinical tasks. Once these recommendations are retrieved, their constraints (in this case, their temporal constraints) are interpreted by the tool and mapped onto the different views mentioned earlier. As an example, considering that the Execution Engine recommends a clinical action to perform a complete physical exam on the patient every 6 months, for 2 years, the application will interpret these restrictions and unfold the recommendation in multiple events and register them in the timeline. As such, the result would be four new events scheduled 6 months apart. Then, the user can consult on the timeline and calendar widgets the scheduling of these events in order to execute the clinical task and so manage better his time.

In order to ensure the execution of tasks at the designated time, it was necessary to implement a notification system and a message box. These elements are both shown in Figure 4. The message box features messages such as indications about the tasks that should be performed or should have already been performed, offering the possibility to mark them as executed. As for the notification system, it is used to periodically alert the user about task enactment times, according to their respective temporal restrictions. The notifications are shown as a pop-up message.

\section{Conclusions and Future Work}

The personal assistant presented herein aims to stretch the reach of CIGs far beyond the medical office. Its purpose as a reminder tool is to ensure the timely enactment of clinical procedures over the course of patient management, removing the possibility of inadvertently skipping steps that may prove to be crucial later on for his recovery. In addition to decision support functionalities, common to other CIG systems, the tool provides additional scheduling and alert features to assist the health care professional in keeping track of their patients. Therefore, its main contribution is a new method to integrate CPG advice in a clinical setting and make it easily available. With the development of the CompGuide personal assistant web application, new questions arose. The first is: how should the delays in task execution be handled? In this case, there are several alternatives, but all of them have drawbacks. Re-scheduling the task may imply verifying if the state of the patient allows the enactment of the procedure at a later time. Not performing the task may be equally damaging to the patient. Another issue is: how should parallel clinical tasks be shown to the health care professional in order to avoid confusion? These are issues we plan to address in future work. 


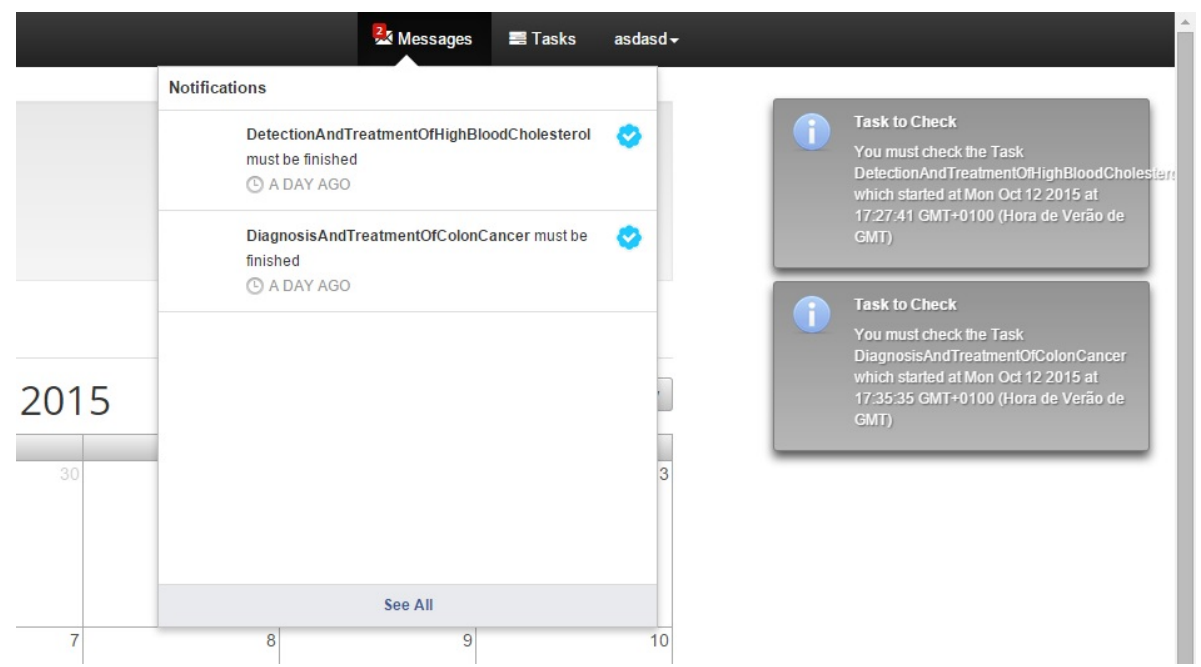

Fig. 4: Message box and notification in the CompGuide personal assistant web application.

\section{Acknowledgements}

This work has been supported by COMPETE: POCI-01-0145-FEDER-0070 43 and FCT Fundação para a Ciência e Tecnologia within the Project Scope UID/CEC/ 00319/2013.

\section{References}

1. Berg, D., Ram, P., Glasgow, J., Castro, J.: SAGEDesktop: An Environment for Testing Clinical Practice Guidelines. The 26th Annual International Conference of the IEEE Engineering in Medicine and Biology Society 4, 3217-3220 (2004)

2. Costa, A., Novais, P., Corchado, J.M., Neves, J.: Increased performance and better patient attendance in an hospital with the use of smart agendas*. Logic Journal of the IGPL 20(4), 689 (2012), 10.1093/jigpal/jzr021

3. Isern, D., Moreno, A.: Computer-based Execution of Clinical Guidelines: a Review. International Journal of Medical Informatics 77(12), 787-808 (2008)

4. Jiang, J., Khelifi, F., Trundle, P., Geven, A.: HERMES: a FP7 funded project towards the development of a computeraided memory management system via intelligent computations. Journal of Assistive Technologies 3(3), 27-35 (sep 2009), http://dx.doi.org/10.1108/17549450200900022

5. Lima, L., Novais, P., Neves, J., Bulas, C.J., Costa, R.: Group Decision Making and Quality-of-Information in e-Health Systems. Logic Journal of the IGPL 19(2), 315-332 (2011)

6. Musen, M.A., Shahar, Y., Shortliffe, E.H.: Clinical decision-support systems. In: Shortliffe, E., Cimino, J. (eds.) Biomedical Informatics, pp. 698-736. Health Informatics, Springer New York (2006) 
7. Novais, P., Costa, R., Carneiro, D., Neves, J.: Inter-organization cooperation for ambient assisted living. J. Ambient Intell. Smart Environ. 2(2), 179-195 (Apr 2010)

8. Oliveira, T., Leão, P., Novais, P., Neves, J.: Webifying the Computerized Execution of Clinical Practice Guidelines. In: Bajo Perez, J., Corchado Rodríguez, J.M., Mathieu, P., Campbell, A., Ortega, A., Adam, E., Navarro, E.M., Ahrndt, S., Moreno, M.N., Julián, V. (eds.) Trends in Practical Applications of Heterogeneous Multi-Agent Systems. The PAAMS Collection SE - 18, Advances in Intelligent Systems and Computing, vol. 293, pp. 149-156. Springer International Publishing (2014)

9. Oliveira, T., Novais, P., Neves, J.: Representation of Clinical Practice Guideline Components in OWL. In: Trends in Practical Applications of Agents and Multiagent Systems SE - 10, Advances in Intelligent Systems and Computing, vol. 221, pp. 77-85. Springer International Publishing (2013)

10. Peleg, M.: Computer-interpretable Clinical Guidelines: A Methodological Review. Journal of Biomedical Informatics 46(4), 744-63 (2013)

11. Picking, R., Robinet, A., Grout, V., McGinn, J., Roy, A., Ellis, S., Oram, D.: A case study using a methodological approach to developing user interfaces for elderly and disabled people. The Computer Journal 53(6), 842 (2010), +http: //dx.doi.org/10.1093/comjnl/bxp089

12. Shalom, E., Shahar, Y., Lunenfeld, E.: An architecture for a continuous, userdriven, and data-driven application of clinical guidelines and its evaluation. Journal of Biomedical Informatics (November) (2015)

13. Terenziani, P., Montani, S., Bottrighi, A., Torchio, M., Molino, G., Correndo, G.: The GLARE Approach to Clinical Guidelines: Main Features. Studies in Health Technology and Informatics 101(3), 162-6 (2004)

14. Wang, D., Peleg, M., Tu, S.W., Boxwala, A.A., Ogunyemi, O., Zeng, Q., Greenes, R.A., Patel, V.L., Shortliffe, E.H.: Design and implementation of the GLIF3 guideline execution engine. Journal of Biomedical Informatics 37(5), 305-318 (2004)

15. Young, O., Shahar, Y.: The Spock System : Developing a Runtime Application Engine for Hybrid-Asbru Guidelines. Artificial Intelligence Review 3581(1), 166$170(2005)$ 